\title{
Synchronization of chaotic systems by using occasional coupling
}

\author{
Ömer Morgül* and Moez Feki* \\ Department of Electrical and Electronics Engineering, Bilkent University, 06533, Bilkent, Ankara, Turkey
}

(Received 24 June 1996; revised manuscript received 18 November 1996)

\begin{abstract}
We present a method for the synchronization of chaotic systems by using occasional coupling. In this method we assume that a chaotic drive system and a response system to be synchronized with the drive system are given. We also assume that a scheme that results in exponentially fast synchronization is available (full synchronization). Then we present an occasional-coupling scheme in which the drive and response systems are coupled in some intervals (synchronization phases) and decoupled in some intervals (autonomous phases), i.e., the response system is switched to an autonomous system. We prove that if the lengths of these intervals are appropriately chosen, then under some mild conditions synchronization can be achieved. We also show that the proposed scheme is robust with respect to noise and parameter mismatch under some mild conditions.
\end{abstract} [S1063-651X(97)03804-X]

PACS number(s): $05.45 .+\mathrm{b}$

\section{INTRODUCTION}

Recently the idea of synchronization of chaotic systems has received a great deal of interest from scientists of various fields $[1-11]$. The configuration we consider for the synchronization of chaotic systems consists of two parts, a generator of chaotic signals (drive system) and a receiver (response system). This configuration is the one most commonly discussed in the literature, due to its possible applications in secure communications. The response system is usually a duplicate of a part (or the whole) of the drive system. A chaotic signal generated by the drive system may be used as an input in the response system to synchronize the common signals of both systems [2]. One motivation for synchronization is the possibility of sending messages through chaotic systems for secure communication (see, e.g., $[5,7,9]$ ).

In this paper we present a synchronization scheme for chaotic systems. As in most synchronization schemes, we assume that a drive and a response system are given. The drive system generates chaotic signals and some of these signals are used in the response system for synchronization. We also assume that a synchronization scheme, for which the synchronization is achieved exponentially fast, is available, i.e., the synchronization error decays exponentially to zero. We note that this requirement is satisfied in many synchronization schemes proposed in the literature $[2,3,8]$. Recently, a synchronization scheme that guarantees this property and is applicable to a broad class of chaotic systems under some mild conditions has been proposed $[12,13]$. The occasional-synchronization scheme proposed in this paper consists of the application of two phases, namely, the synchronization and autonomous phases continually following each other. In the synchronization phases, the exponential synchronization scheme mentioned above is used to synchronize the drive and the response systems and in the autonomous phases the response system is switched to an autonomous system. We show that if the synchronization and the

\footnotetext{
*Fax: 90-312-266 41 26. Electronic address: morgul@bilkent.edu.tr
}

autonomous phase intervals are chosen appropriately, then the synchronization can be achieved exponentially fast in the ideal case (i.e., when the synchronization link is not corrupted with noise and parameters of drive and the response systems are known exactly). We also show that the proposed scheme is robust with respect to noise in the synchronization link and parameter mismatch, i.e., the synchronization error remains bounded, and this bound decreases to zero as the noise and the parameter mismatch magnitudes decrease to zero.

There may be various reasons for using autonomous phases. As mentioned in Ref. [11], in some cases it may be impossible to use only synchronization at all times and, moreover, to use synchronization in some intervals only may prove to be more cost effective than using synchronization at all times. Another reason might be the possibility of sending chaotically masked or coded messages for communication in the autonomous phases [14].

We note that a related idea for synchronization of chaotic systems was proposed in Ref. [11], where drive variables are not used in the response system at all times. Instead, for a finite time step $\tau$ at instances $t=n \tau$, for $n=0,1.2, \ldots$, the response system states corresponding to the drive variables used for synchronization are set to the values of the corresponding drive system variables, and it was shown that, for sufficiently small values of $\tau$, synchronization is possible. Moreover, in the limit of $\tau \rightarrow 0$, this method reduces to the method proposed in Ref. [2]. Hence, in the scheme proposed in Ref. [11], the synchronization is achieved at discrete times (i.e., not in a time interval), whereas in our scheme the synchronization is achieved in an interval. We note that the length of this interval is of crucial importance for the stability analysis in our scheme. Both of these schemes use an autonomous phase interval, and as in the scheme proposed in Ref. [11], when only a synchronization phase is used (i.e., no autonomous phase), our scheme reduces to the scheme proposed in Ref. [2]. There are other schemes that use different occasional coupling for synchronization of chaotic systems, see Ref. [15] and the references therein.

This paper is organized as follows. In Sec. II we introduce our scheme and prove that under some mild conditions, in 
the ideal case (i.e., noise is not present and the parameters of drive and response systems match), exponential synchronization can be achieved, provided that the lengths of the synchronization and the autonomous phase intervals are chosen accordingly. We then consider the nonideal case and prove that the proposed scheme is robust with respect to noise and parameter mismatch under some mild conditions, provided that the synchronization and autonomous phase intervals are chosen appropriately. In this case the synchronization error is bounded, and the bound depends linearly on the magnitude of the noise and the parameter mismatch. Then we present some simulation results, and finally give some concluding remarks.

\section{OCCASIONAL COUPLING IN THE IDEAL CASE}

Assume that the drive system is given as follows:

$$
\dot{u}=f(u, \mu), \quad u \in \mathbf{R}^{n}, \quad \mu \in \mathbf{R}^{p},
$$

where $f(\cdot): \mathbf{R}^{n} \times \mathbf{R}^{p} \rightarrow \mathbf{R}^{n}$ is a differentiable function, $\mu \in \mathbf{R}^{p}$ is a parameter vector. We assume that $\mu$ is such that system (1) exhibits chaotic behavior. In the response system, some signals generated by Eq. (1) will be used for synchronization. To simplify the notation, let us define an "output" $o$ corresponding to the system given by Eq. (1) as follows:

$$
o=h(u), \quad u \in \mathbf{R}^{n}, \quad o \in \mathbf{R}^{m},
$$

where $h(\cdot): \mathbf{R}^{n} \rightarrow \mathbf{R}^{m}$ is a differentiable function. For the response system we consider the following:

$$
\dot{w}=g(o, w, \mu), \quad w \in \mathbf{R}^{n},
$$

where $g(\cdot): \mathbf{R}^{m} \times \mathbf{R}^{n} \times \mathbf{R}^{p} \rightarrow \mathbf{R}^{n}$ is a differentiable function. Note that Eq. (3) signifies the fact that some of the signals of the drive system are used for synchronization in the response system.

For the drive and the response systems given above, we assume that the following conditions hold.

Assumption 1: The following Lipschitz condition is satisfied:

$$
\|f(u, \mu)-f(w, \mu)\| \leqslant k\|u-w\|, \quad u, w \in \mathbf{R}^{n}, \quad \mu \in \mathbf{R}^{p},
$$

where $k>0$ is a Lipschitz constant and the norm $\|\cdot\|$ is the standard Euclidean norm.

Assumption 2: The following is satisfied:

$$
g(h(w), w, \mu)=f(w, \mu), \quad w \in \mathbf{R}^{n}, \quad \mu \in \mathbf{R}^{p} .
$$

Assumption 3: The drive system given by Eq. (1) and the response system given by Eq. (3) are exponentially synchronized, i.e., there exist constants $M>0$ and $\delta>0$, such that for any initial time $t_{0}$ and for any initial conditions $u\left(t_{0}\right), w\left(t_{0}\right) \in \mathbf{R}^{n}$, the following is satisfied:

$$
\|u(t)-w(t)\| \leqslant M e^{-\alpha\left(t-t_{0}\right)}\left\|u\left(t_{0}\right)-w\left(t_{0}\right)\right\| .
$$

Remark 1: The Lipschitz condition given by Eq. (4) might seem restrictive. However, since $f(\cdot)$ is differentiable, Eq. (4) is satisfied in any compact ball, i.e., for any $\epsilon_{r}>0$, Eq. (4) is satisfied for $\|u\| \leqslant \epsilon_{r}$ and $\|w\| \leqslant \epsilon_{r}$. If we assume that the drive system is chaotic, then the solutions of Eq. (1), that are of interest to us, are bounded in a region and, hence, in this region Eq. (4) is satisfied.

Assumption 2 is not very restrictive and is satisfied in many synchronization schemes proposed in the literature $[1,3,8]$.

Assumption 3 might seem restrictive. However, this condition is also satisfied in many synchronization schemes proposed in the literature $[1,3,8]$. Recently, a general synchronization scheme that guarantees exponential synchronization under some mild conditions, and is applicable to a broad class of chaotic systems, has been proposed $[12,13]$.

We now state our occasional-synchronization scheme. Let the intervals $T_{s}>0$ and $T_{a}>0$ denote the occasionalsynchronization and autonomous phase intervals, respectively. Our scheme is as follows $(i=1,2, \ldots)$.

(i) (ith occasional-synchronization phase). For $(i-1)\left(T_{s}+T_{a}\right) \leqslant t<i T_{s}+(i-1) T_{a}$, use the drive system given by Eq. (1) and the response system given by Eq. (3).

(ii) (ith autonomous phase). For $i T_{s}+(i-1) T_{a} \leqslant t$ $<i\left(T_{s}+T_{a}\right)$ use the drive system given by Eq. (1), and for the response system use the following:

$$
\dot{w}=g(h(w), w, \mu)=f(w, \mu) .
$$

Hence, in our scheme, occasional-synchronization and autonomous phases follow each other. Note that with Eq. (7) the response system becomes an autonomous system in the autonomous phase. Since, in the synchronization phase, the error decays to zero exponentially fast [see Eq. (6)], at the end of this phase the error becomes extremely small, provided that $T_{s}$ is sufficiently large. Hence, in the autonomous phase we could switch the signals of the drive system used for synchronization with the corresponding signals of the response system, which is the rationale behind using Eq. (7) instead of Eq. (3).

At this point, we compare our scheme with that of [2] and [11]. If we choose $T_{s}=0$ (i.e., the synchronization phase interval does not exist), set $T_{a}=\tau$, and use the synchronization at discrete times $n \tau, n=0,1, \ldots$, then the scheme presented above reduces to that of [11]. On the other hand, if we choose $T_{a}=0$ (i.e., the autonomous phase interval does not exist) and use only the synchronization phase (i.e., $T_{s}=\infty$ ), then the scheme presented above reduces to that of [2]. We note that the nonzero length of $T_{s}>0$ is of crucial importance for our stability analysis (see Theorem 1, Eqs. (14) and (15), below).

For simplicity, we define the beginning of $i$ th synchronization and autonomous phases $T_{i}^{s}$ and $T_{i}^{a}$, respectively, as follows:

$$
T_{i}^{s}=(i-1)\left(T_{s}+T_{a}\right), \quad T_{i}^{a}=i T_{s}+(i-1) T_{a}, \quad i=1,2, \ldots
$$

We also define the synchronization error $e(t)$ as follows:

$$
e(t)=u(t)-w(t) .
$$

From Eq. (6) it is clear that the following holds in the $i$ th occasional-synchronization phase:

$$
\|e(t)\| \leqslant M e^{-\alpha\left(t-T_{i}^{s}\right)}\left\|e\left(T_{i}^{s}\right)\right\|, \quad T_{i}^{s} \leqslant t<T_{i}^{a} .
$$


From Eqs. (1) and (7) it follows that the following holds in the $i$ th autonomous phase:

$$
\begin{array}{r}
e(t)=e\left(T_{i}^{a}\right)+\int_{T_{i}^{a}}^{t}[f(u(\tau), \mu)-f(w(\tau), \mu)] d \tau, \\
T_{i}^{a} \leqslant t<T_{i+1}^{s} .
\end{array}
$$

By using Eq. (4), taking norms, and using the BellmanGronwall lemma [16], we obtain

$$
\|e(t)\| \leqslant e^{k\left(t-T_{i}^{a}\right)}\left\|e\left(T_{i}^{a}\right)\right\|, \quad T_{i}^{a} \leqslant t<T_{i+1}^{s} .
$$

By using Eqs. (12) and (10) successively, and noting that the error is continuous at switching instances $T_{i}^{s}$ and $T_{i}^{a}$, we obtain

$$
\begin{aligned}
& \|e(t)\| \leqslant e^{k T_{a}}\left\|e\left(T_{i}^{a}\right)\right\|, \quad T_{i}^{a} \leqslant t<T_{i+1}^{s} \\
& \leqslant M e^{\left(k T_{a}-\alpha T_{s}\right)}\left\|e\left(T_{i}^{s}\right)\right\| \\
& \leqslant\left(M e^{\left(k T_{a}-\alpha T_{s}\right)}\right)^{i}\|e(0)\| .
\end{aligned}
$$

Now we state our first result.

Theorem 1: Let Assumptions 1-3 be satisfied and consider the occasional-synchronization scheme presented above. Let the synchronization and autonomous phase intervals $T_{s}$ and $T_{a}$ be chosen as

$$
\begin{gathered}
T_{s}>\frac{\ln M}{\alpha}, \\
T_{a}<\frac{\alpha T_{s}-\ln M}{k},
\end{gathered}
$$

then, for any initial error $e(0)$, the synchronization error decays to zero asymptotically, i.e., $\lim _{t \rightarrow \infty}\|e(t)\|=0$. Moreover, the decay is exponential.

Proof: From Eq. (13) it is obvious that, if

$$
M e^{\left(k T_{a}-\alpha T_{s}\right)}<1,
$$

then we have $\left.\| e\left(T_{i+1}^{s}\right)\right)\|<\| e\left(T_{i}^{s}\right) \|$. By using Eq. (16), we obtain Eq. (15). To guarantee that $T_{a}>0$, we need $\alpha T_{s}-\ln M>0$; hence, Eq. (14) follows. From Eq. (14) we

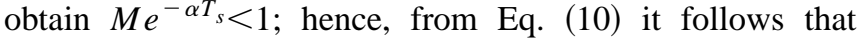
$\left\|e\left(T_{i}^{a}\right)\right\|<\left\|e\left(T_{i}^{s}\right)\right\|$. By using this result Eqs. (13) and (16), it follows that $\lim _{t \rightarrow \infty}\|e(t)\|=0$.

For exponential decay, note that Eq. (13) is valid for $T_{i}^{a} \leqslant t<T_{i+1}^{s}$, which implies that $t /\left(T_{a}+T_{s}\right)<i$. Let us define $\rho=M e^{\left(k T_{a}-\alpha T_{s}\right)}$. From Eq. (13) we obtain

$$
\|e(t)\| \leqslant\left(\frac{1}{\rho}\right)^{-i}\|e(0)\| \leqslant e^{-\lambda t}\|e(0)\|, \quad T_{i}^{a} \leqslant t<T_{i+1}^{s},
$$

where $\lambda=-\ln \rho /\left(T_{a}+T_{s}\right)$. Note that, since $\rho<1$ [see Eq. (16)], we have $\lambda>0$. By using this result and Eq. (10), it follows that the error decays exponentially to zero.

Remark 2: Theorem 1 does not imply that the error continually decreases to zero. In fact, in autonomous phases, obviously the error increases. However, since $\left\|e\left(T_{i+1}^{s}\right)\right\|<\left\|e\left(T_{i}^{s}\right)\right\|$, it follows that the error at the end of an autonomous phase is strictly less than the error at the begin- ning of preceding synchronization phase. Basically, for this reason we can find an exponentially decaying function that bounds the error.

Remark 3: In the development presented above we assumed that the interval lengths $T_{s}$ and $T_{a}$ are the same in each synchronization and autonomous phases. However, we could choose different interval lengths in each synchronization and autonomous phases and the results of Theorem 1 will be valid, provided that Eqs. (14) and (15) are satisfied.

The development presented above could be used to derive further results. For example, assume that the initial error satisfies $\|e(0)\| \leqslant \epsilon_{r}$ for some $\epsilon_{r}>0$, and it is required that the error satisfy $\|e(t)\| \leqslant \epsilon$ in $i$ th autonomous phase, where $\epsilon>0$ is a given precision level. From Eq. (13) it follows that this requirement can be satisfied, provided that the interval lengths $T_{s}$ and $T_{a}$ are chosen as follows:

$$
\begin{gathered}
T_{s}>\frac{\ln M+(1 / i) \ln \epsilon_{r} / \epsilon}{\alpha}, \\
T_{a}<\frac{\alpha T_{s}-\ln M-(1 / i) \ln \epsilon_{r} / \epsilon}{k} .
\end{gathered}
$$

Note that, if we take the limit when $i \rightarrow \infty$, Eqs. (17) and (18) reduce to Eqs. (14) and (15).

\section{ROBUSTNESS WITH RESPECT TO NOISE AND PARAMETER MISMATCH}

Consider the drive and the response systems given by Eqs. (1) and (3), respectively. Because of the exponential synchronization assumption (5), we expect that for the dynamical system governing the behavior of the error (i.e., error dynamics), $e=0$ is an exponentially stable equilibrium point. We note that this assumption holds in most of the synchronization schemes proposed in the literature $[2,6,8]$. Since exponentially stable systems are robust with respect to small perturbations in the dynamics, and since our scheme yields exponentially fast synchronization in the ideal case, we expect that the synchronization scheme proposed here is also robust with respect to small perturbations [17], pp. 191209.

To justify the robustness properties analytically, we need to specify the error dynamics. First, let us assume the ideal case, i.e., noise is not present and the parameters match exactly. For simplicity we assume that the error dynamics is as given below

$$
\dot{e}=F(o, u, e, \mu),
$$

where $F$ is a differentiable function of its arguments, $o, u$, and $\mu$ are as defined before. We note that the form of the error dynamics given by Eq. (19) is not the only possible form to conclude the robustness results. We choose this form because it could be expected from Eqs. (1) and (3) and, for the synchronization schemes given in Refs. [2,6,8], the error dynamics can be put in the form given by Eq. (19). Note that $u$ can be considered an exogenous signal for the error dynamics; hence, we can view Eq. (19) as a time-varying system.

Since we assume exponential synchronization, it follows that $e=0$ is an exponentially stable equilibrium point of Eq. 
(19). Hence, by a well-known result in the Lyapunov stability theory, there exists a Lyapunov function $V: \mathbf{R} \times \mathbf{R}^{n} \rightarrow \mathbf{R}$, which satisfies the following:

$$
\begin{gathered}
c_{1}\|e\|^{2} \leqslant V(t, e) \leqslant c_{2}\|e\|^{2}, \\
\dot{V}=\frac{\partial V}{\partial t}+\frac{\partial V}{\partial e} F \leqslant-c_{3}\|e\|^{2}, \\
\left\|\frac{\partial V}{\partial e}\right\| \leqslant c_{4}\|e\|,
\end{gathered}
$$

for some positive constants $c_{1}, c_{2}, c_{3}, c_{4}[17,18]$. We note that the existence of such a Lyapunov function is both necessary and sufficient for exponential stability, see [17], p. 180. Moreover, the constants in Eq. (6) can be given as $M$ $=\sqrt{c_{2} / c_{1}}$ and $\alpha=c_{3} / 2 c_{2}$.

In the nonideal case, the occasional synchronization scheme presented in the preceding section takes the following form.

(i) (ith occasional-synchronization phase). For $T_{i}^{s} \leqslant t<T_{i}^{a}$, the drive system is given by Eq. (1) and the response system is given by the following [cf. Eq. (3)]:

$$
\dot{w}=g\left(o+n, w, \mu^{\prime}\right),
$$

where $o$ is the output of the drive system given by Eq. (2), $n$ represents the noise acting on the output, and $\mu^{\prime}$ is the parameter vector of the response system. We note that the noise could be an arbitrary function of time.

(ii) (ith autonomous phase). For $T_{i}^{a} \leqslant t<T_{i+1}^{s}$, the drive system is given by Eq. (1) and the response system is switched to an autonomous system. Therefore, in this phase, the drive system is not affected by the noise in the synchronization link and is given by [cf. Eq. (7)]

$$
\dot{w}=f\left(w, \mu^{\prime}\right) \text {. }
$$

For the robustness analysis, we need the following assumptions:

Assumption 4: The following Lipschitz conditions are satisfied for some positive constants $k_{1}, k_{2}, k_{3}$ :

$$
\begin{gathered}
\left\|g\left(o_{1}, w, \mu\right)-g\left(o_{2}, w, \mu\right)\right\| \leqslant k_{1}\left\|o_{1}-o_{2}\right\|, \\
o_{1}, o_{2} \in \mathbf{R}^{m}, \quad w \in \mathbf{R}^{n}, \quad \mu \in \mathbf{R}^{p}, \quad(25) \\
\left\|g(o, w, \mu)-g\left(o, w, \mu^{\prime}\right)\right\| \leqslant k_{2}\left\|\mu-\mu^{\prime}\right\|, \\
o \in \mathbf{R}^{m}, \quad w \in \mathbf{R}^{n}, \quad \mu, \mu^{\prime} \in \mathbf{R}^{p}, \quad(26) \\
\left\|f(u, \mu)-f\left(u, \mu^{\prime}\right)\right\| \leqslant k_{3}\left\|\mu-\mu^{\prime}\right\|, \quad u \in \mathbf{R}^{n}, \quad \mu, \mu^{\prime} \in \mathbf{R}^{p},
\end{gathered}
$$

where $\|\cdot\|$ represents the standard Euclidean norm in $\mathbf{R}^{n}, \mathbf{R}^{m}$, or $\mathbf{R}^{p}$.

We note that these requirements are not very restrictive. Since we assume that the signals are chaotic, and therefore bounded, Eqs. (25)-(27) may be considered a consequence of differentiability of $g$ with respect to its arguments.

\section{A. Robustness in the occasional synchronization phase}

By using Eqs. (1) and (3) we obtain $F(o, u, e, \mu)=f(u, \mu)$ $-g(o, w, \mu)$ in the ideal case. Hence, by using Eqs. (19) and (23) we obtain the following error dynamics for the nonideal case:

$$
\begin{aligned}
\dot{e}= & F(o, u, e, \mu)+\left[g(o, w, \mu)-g\left(o, w, \mu^{\prime}\right)\right] \\
& +\left[g\left(o, w, \mu^{\prime}\right)-g\left(o+n, w, \mu^{\prime}\right)\right] .
\end{aligned}
$$

In the ideal case we have $n=0$ and $\mu=\mu^{\prime}$, and Eq. (28) reduces to Eq. (19). Since the latter is exponentially stable, the terms in square brackets in Eq. (28) represent perturbations to an exponentially stable system. By using the exponential stability of the error dynamics in the ideal case, we can now prove the following robustness result for Eq. (28).

Theorem 2: Consider the error dynamics given by Eq. (28). Assume that Eqs. (25) and (26) hold. Let the noise $n$ satisfy $\|n(t)\| \leqslant n_{m}$ for some $n_{m}>0$ for $t \geqslant 0$ and let us define $\Delta \mu=\mu-\mu^{\prime}$. Then the error asymptotically (i.e., as $t \rightarrow \infty$ ) satisfies the following inequality:

$$
\|e(t)\| \leqslant C_{1} n_{m}+C_{2}\|\Delta \mu\|,
$$

where $C_{1}>0$ and $C_{2}>0$ are some constants.

Proof: Let us consider the Lyapunov function which satisfies Eqs. (20)-(22). Since the error dynamics is exponentially stable in the ideal case, such a function always exists. By differentiating $V$ along the solutions of Eq. (28), we obtain

$$
\begin{aligned}
\dot{V}= & \frac{\partial V}{\partial t}+\frac{\partial V}{\partial e} F+\frac{\partial V}{\partial e}\left[g(o, w, \mu)-g\left(o, w, \mu^{\prime}\right)\right] \\
& +\frac{\partial V}{\partial e}\left[g\left(o, w, \mu^{\prime}\right)-g\left(o+n, w, \mu^{\prime}\right)\right] \\
\leqslant & -c_{3}\|e\|\left[\|e\|-\frac{c_{4}}{c_{3}}\left(k_{1} n_{m}+k_{2}\|\Delta \mu\|\right)\right]
\end{aligned}
$$

where we used Eqs. (21), (22), (25), and (26). From Eq. (30) it follows that if $\|e\|>\left(c_{4} / c_{3}\right)\left(k_{1} n_{m}+k_{2}\|\Delta \mu\|\right)$, then $\dot{V}<0$ and, hence, $V$ and, by Eq. (20), error $e$ decrease along the solutions of Eq. (28). It then follows from the standard invariance arguments that asymptotically Eq. (29) is satisfied [17, p. 187, Theorem 4.8]. In particular, we could choose $C_{1}>\left(c_{4} / c_{3}\right) k_{1}$ and $C_{2}>\left(c_{4} / c_{3}\right) k_{2}$, where $c_{3}$ and $c_{4}$ are given by Eqs. (21) and (22), respectively.

Remark 4: It follows from Eq. (29) that if $n_{m}$ and $\|\Delta \mu\|$ are sufficiently small, then the error will be asymptotically small (cf. Theorem 1). Hence, we can conclude that synchronization schemes for which the error dynamics is exponentially stable, is also robust with respect to noise and parameter mismatch. We note that this result is the basic reason for the robustness of many schemes proposed for synchronization in the literature. Hence, we can view Theorem 2 not only as a result related to the synchronization scheme proposed here, but also as a general result related to any synchronization scheme, provided that its assumptions are satisfied. We also note that the above result is only asymptotic in nature and the required synchronization length $T_{s}$ cannot be specified in general. To estimate the required $T_{s}$, further 
assumptions on the form of the error dynamics given by Eq. (19) may be necessary. For example, in [12] and [13], the following chaotic drive systems are considered:

$$
\dot{u}=\mathbf{A}(\mu) u+r(u, \mu),
$$

where, for a fixed $\mu \in \mathbf{R}^{p}, A(\mu) \in \mathbf{R}^{n \times n}$ is a constant matrix and $r: \mathbf{R}^{n} \times \mathbf{R}^{p} \rightarrow \mathbf{R}^{n}$ is a differentiable function. For this system, the output $o$ is chosen as $o=C u$, where $C \in \mathbf{R}^{m \times n}$ is a constant matrix. Then the response system is chosen as

$$
\dot{w}=A(\mu) w+r(w, \mu)+K(o-C w),
$$

where $K \in \mathbf{R}^{n \times m}$ is a constant gain matrix to be determined. This scheme is called observed based synchronization and it was shown in [12] and [13] that, for a wide class of chaotic systems, this scheme yields exponentially fast synchronization under some mild conditions. In this case, the error dynamics is given by [cf. Eq. (19)]

$$
\dot{e}=[A(\mu)-K C] e+r(u, \mu)-r(w, \mu) .
$$

By an appropriate choice of the gain matrix $K$, it could be shown that the error decays exponentially to zero, i.e., Eq. (6) is satisfied for some $M>0$ and $\alpha>0$. In the nonideal case, it was shown in [13] that the error satisfies the following:

$$
\|e(t)\| \leqslant C\left(1-e^{-\alpha t}\right)+M e^{-\alpha t}\|e(0)\|, \quad t \geqslant 0,
$$

where $C=A n_{M}+B\|\Delta \mu\|$ for some positive constants $A$ and $B$. By comparing Eqs. (34) and (29), we see that asymptotically the latter is satisfied with $A=\left(c_{4} k_{1} / c_{3}\right), B=\left(c_{4} k_{2} / c_{3}\right)$. From Eqs. (34) and (29) it follows that the latter is satisfied as $t \rightarrow \infty$. Assuming that Eq. (34) holds, we could estimate the required synchronization length $T_{s}$ in order to bound the error by a given precision level $\epsilon_{s}$. Let such a level $\epsilon_{s}>0$ be given and let the initial error satisfy $\|e(0)\| \leqslant \epsilon_{r}$ for some $\epsilon_{r}>0$. Then it follows from Eq. (34) that we have $\|e(t)\| \leqslant \epsilon_{s}$ for $t \geqslant T$ where

$$
T \geqslant \frac{1}{\alpha} \ln \left(\frac{M \epsilon_{r}-C}{\epsilon_{s}-C}\right) .
$$

For Eq. (35) to be meaningful, we need $\epsilon_{s}>C$. Hence, the required synchronization length should satisfy $T_{s} \geqslant T$. Note that in the ideal case (i.e., $n_{M}=0, \Delta \mu=0$ ), if we choose $\epsilon_{s}=\epsilon_{r}$ (i.e., $M e^{-\alpha T_{s}<1}$, see Theorem 1), then Eq. (35) reduces to Eq. (14).

\section{B. Robustness in the autonomous phase}

By using Eqs. (1) and (24), we obtain the following in the autonomous phase:

$$
\begin{aligned}
e(t)= & e\left(T_{s}\right)+\int_{T_{s}}^{t}[f(u(\tau), \mu)-f(w(\tau), \mu)] d \tau \\
& +\int_{T_{s}}^{t}\left[f(w(\tau), \mu)-f\left(w(\tau), \mu^{\prime}\right)\right] d \tau, \quad t \geqslant T_{s} .
\end{aligned}
$$

By taking norms in Eq. (36), and by using Eqs. (4) and (27), we obtain

$$
\begin{aligned}
&\|e(t)\| \leqslant\left\|e\left(T_{s}\right)\right\|+k_{3}\|\Delta \mu\|\left(t-T_{s}\right)+\int_{T_{s}}^{t} k\|e(\tau)\| d \tau, \\
& t \geqslant T_{s} .
\end{aligned}
$$

Now assume that the autonomous phase takes place in the interval $T_{s} \leqslant t<T_{s}+T_{a}$. Then, by using the BellmanGronwall inequality, we obtain

$$
\|e(t)\| \leqslant\left(\left\|e\left(T_{s}\right)\right\|+k_{3}\|\Delta \mu\| T_{a}\right) e^{k T_{a}}, \quad T_{s} \leqslant t<T_{s}+T_{a} .
$$

Now assume that $\left\|e\left(T_{s}\right)\right\| \leqslant \epsilon_{s}$ and we require $\|e(t)\| \leqslant \epsilon_{a}$ for $T_{s} \leqslant t<T_{s}+T_{a}$ for some precision levels $\epsilon_{s}>0, \epsilon_{a}>0$. Obviously we should have $\epsilon_{a}>\epsilon_{s}$. Then, from Eq. (37) it follows that $T_{a}$ should satisfy

$$
e^{k T_{a}<\frac{\epsilon_{a}}{\epsilon_{s}+k_{3}\|\Delta \mu\| T_{a}} .}
$$

There exists a $T>0$ such that Eq. (38) is satisfied for all $T_{a} \leqslant T$. To see that, note that Eq. (38) is satisfied for $T_{a}=0$. Since the left and right sides of Eq. (38) are strictly increasing and decreasing functions of $T_{a}$, respectively, it follows easily that such a $T>0$ exists.

Note that in the ideal case we have $\Delta \mu=0$ and $\epsilon_{s}$ $=M e^{-\alpha T_{s}} \epsilon_{r}$, where $\|e(0)\| \leqslant \epsilon_{r}$ [see Eq. (6)]. If $T_{s}$ satisfies

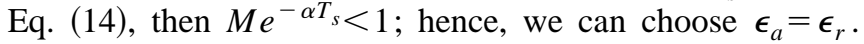
With this choice, Eq. (38) reduces to Eq. (15) in the ideal case.

From the analysis presented above, it is clear that if we choose $T_{s}$ sufficiently large, $T_{a}$ sufficiently small, and apply our occasional synchronization scheme, it is possible to keep the error below a reasonable precision level. From a practical point of view, $T_{s}$ and $T_{a}$ should be chosen sufficiently larger and smaller than the bounds given by Eqs. (14) and (15), respectively.

\section{SIMULATION RESULTS}

For an application of the ideas given above, we consider the well-known Lorenz system for the drive system [2],

$$
\begin{gathered}
\dot{x}=\sigma(y-x), \\
\dot{y}=-x z+r x-y, \\
\dot{z}=x y-b z .
\end{gathered}
$$

We choose the parameters $\sigma, r$, and $b$, so that the system (39) is in the chaotic regime as $\sigma=10, r=20, b=1$. We note that Eq. (39) is in the form of Eq. (1). The solution $x(t)$ of Eq. (39) will be used to synchronize the solutions of the following response system [8]:

$$
\begin{gathered}
\dot{x}_{r}=\sigma\left(y_{r}-x_{r}\right), \\
\dot{y}_{r}=-x z_{r}+r x-y_{r}, \\
\dot{z}_{r}=x y_{r}-b z_{r} .
\end{gathered}
$$

In our notation we have $o=x$, hence $m=1$, and, with $u=(x y z)^{T}$, we have $o=C u$ with $C=(100)$. Also note that 
the response system (40) is of the form given by Eq. (3), moreover, Eqs. (4) and (5) are satisfied. It was shown in Ref. [8] that the signals of Eqs. (39) and (40) are asymptotically synchronized (see also Ref. [2]). However, here we emphasize that the synchronization is in fact exponential, which is important for the scheme proposed in this work. Let the error terms be defined as $e_{x}=x-x_{r}, e_{y}=y-y_{r}$, and $e_{z}=z-z_{r}$. From Eqs. (39) and (40), we obtain the following error dynamics:

$$
\begin{gathered}
\dot{e}_{x}=-\sigma e_{x}+\sigma e_{y}, \\
\dot{e}_{y}=-e_{y}-x e_{z}, \\
\dot{e}_{z}=-b e_{z}+x e_{y} .
\end{gathered}
$$

Let us choose the following Lyapunov function,

$$
V=\frac{1}{2}\left(\gamma e_{x}^{2}+\rho e_{y}^{2}+\rho e_{z}^{2}\right),
$$

where $\gamma>0$ and $\rho>0$ are constants to be determined. By differentiating Eq. (42) along Eq. (41) and using the simple fact $2 p q \leqslant p^{2}+q^{2}$ for any $p, q \in \mathbf{R}$, we obtain

$$
\dot{V} \leqslant-\frac{\sigma \gamma}{2} e_{x}^{2}-\frac{2 \rho-\gamma \sigma}{2} e_{y}^{2}-\rho b e_{z}^{2} .
$$

By choosing $\rho>0$ and $\gamma>0$ such that $2 \rho>\gamma \sigma$, it follows that Eqs. (20)-(22) are satisfied with $c_{1}=\frac{1}{2} \min \{\gamma, \rho\}$, $c_{2}=\frac{1}{2} \max \{\gamma, \rho\}, \quad c_{3}=\frac{1}{2} \min \{\sigma \gamma, 2 \rho-\gamma \sigma, 2 \rho b\}, \quad c_{4}=\max \{\gamma, \rho\}$. Hence, Eq. (6) is satisfied with $M=\sqrt{c_{2} / c_{1}}$ and $a=c_{3} / 2 c_{2}$.

Since the function $f$ given by Eqs. (1) and (39) is differentiable, it follows that Eq. (4) is satisfied in any compact region in state space. In particular, we have $k \leqslant \sup \{\| \partial f \mid$ $\partial u\|\mid\| u \| \leqslant r\}$ for any $r>0$.

Next we present some numerical simulation results that indicate that the suggested method can be used for successful synchronization. Since the state variables in Eq. (39) vary in a wide dynamical range, for simulation purposes following Ref. [8], we use the scaling $x / 10, y / 10$, and $z / 20$, which results in the following "scaled" Lorenz system:

$$
\begin{gathered}
\dot{x}=\sigma(y-x), \\
\dot{y}=-20 x z+r x-y, \\
\dot{z}=5 x y-b z,
\end{gathered}
$$

and we changed the response system (40) accordingly. In the simulations, we use the SIMULAB software package. We first estimated the bounds given by Eqs. (14) and (15). By using $\gamma=1$ and $\rho=6$ in Eq. (42), we obtained $M=2.44$ and $\alpha=0.16$. Also, by using a typical simulation result of Eq. (39) and by evaluating the associated Jacobian matrix, we estimated the Lipschitz constant in Eq. (4) as $k=18.64$. By using these constants in Eq. (14) we found that $T_{s} \geqslant 5.35$ is required, and, if we choose $T_{s}=15$, from Eq. (15) we found that $T_{a} \leqslant 0.08$ is required. However, in our simulations we were able to obtain longer autonomous phase intervals. This shows that the estimates given in Eqs. (14) and (15) might be quite conservative.

In the first set of simulations, we considered the ideal case and chose $T_{s}=15$ and $T_{a}=18$. As for the initial conditions,
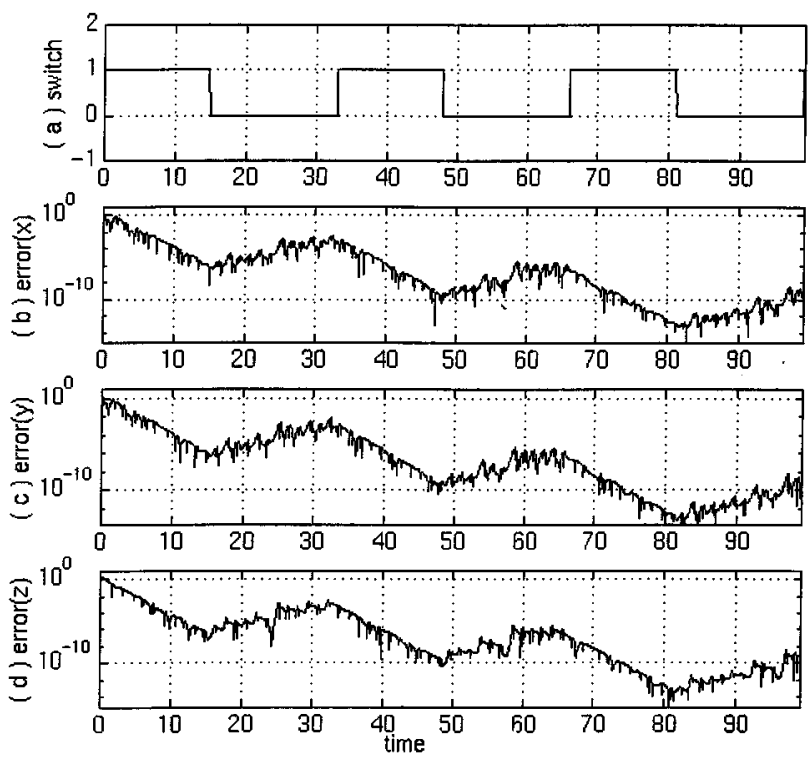

FIG. 1. Master-slave synchronization of the Lorenz system, ideal case. (a) The switch alternates between the synchronization phase and the autonomous phase. (b) Evolution of the error magnitude $\left|e_{x}\right|$. (c) Evolution of the error magnitude $\left|e_{y}\right|$. (d) Evolution of the error magnitude $\left|e_{z}\right|$.

we chose $x(0)=0.8, y(0)=0.1, z(0)=2$ for the drive system, and we chose 0 initial conditions in the response system. The results of the simulation are shown in Fig. 1. Figure 1(a) shows the synchronization and autonomous phase intervals. On the synchronization phase intervals the switch value is equal to 1 , and on the autonomous phase intervals, the switch value is equal to 0 . Figures $1(\mathrm{~b})-1(\mathrm{~d})$ show the evolution of the magnitude of the errors $e_{x}, e_{y}$, and $e_{z}$, respectively. We note that, since the errors are extremely small, it was not possible to obtain meaningful figures on a linear scale, hence we used logarithmic vertical scales in these figures. It is clear from these figures that the errors asymptotically decrease to zero.

In the second set of simulations we considered the nonideal case and chose $T_{s}=15$ and $T_{a}=9$. As for the initial conditions, we chose $x(0)=0.8, y(0)=0.1, z(0)=2$ for the drive system, and we chose 0 initial conditions in the response system. The parameters in the drive system are chosen as $\sigma=10, r=20, b=1$ and in the response system as $\sigma^{\prime}=10.01, r^{\prime}=20.02, b^{\prime}=1.001$, which corresponds to $1 \%$ change in the parameters. We also added a white noise, generated by the computer, to the synchronization signal used in the response system [see Eqs. (23) and (40)]; the magnitude of the white noise is bounded by $n_{M}=10^{-4}$. The results of the simulation are shown in Fig. 2. Figures 2(b)-2(d) show the evolution of the magnitude of the errors $e_{x}, e_{y}$, and $e_{z}$, respectively. As explained above, we used logarithmic vertical scales in these figures. As can be seen from these figures, the errors remain bounded, and the bound on the error is comparable to the noise level.

\section{CONCLUSION}

In this paper, we presented a scheme for the synchronization of chaotic systems by using occasional coupling. As in 

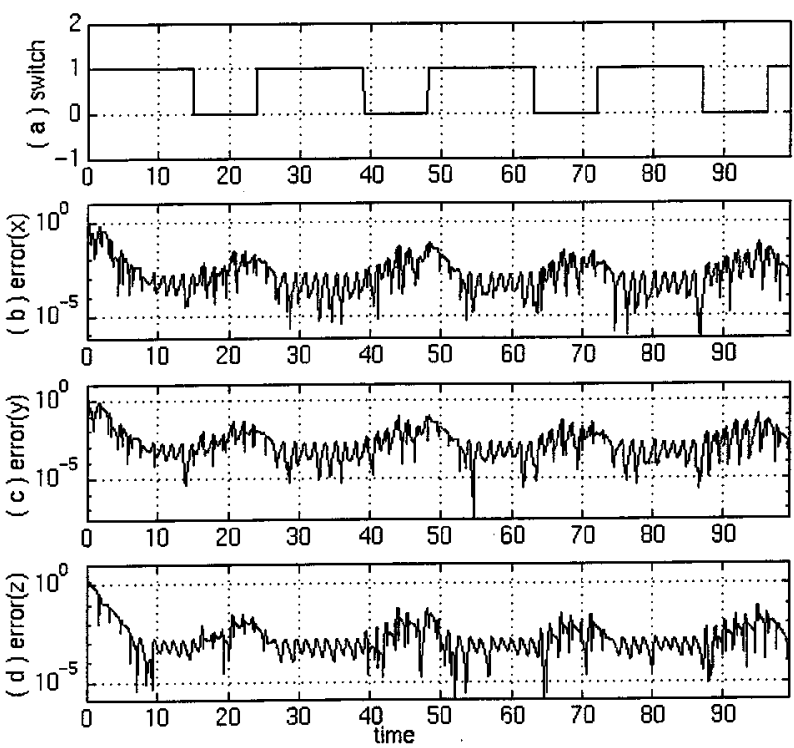

FIG. 2. Master-slave synchronization of the Lorenz system, in the presence of noise and parameter mismatch. (a) The switch alternates between the synchronization phase and the autonomous phase. (b) Evolution of the error magnitude $\left|e_{x}\right|$. (c) Evolution of the error magnitude $\left|e_{y}\right|$. (d) Evolution of the error magnitude $\left|e_{z}\right|$.

most synchronization schemes, we assume that a drive system generates chaotic signals and some of these signals are used in the response system for synchronization. Our scheme consists of the application of two phases, namely, synchronization and autonomous phases continually following each other, and, while the drive and the response systems are synchronized in the synchronization phases, in the autonomous phases the response system is switched to an autonomous system. We assume that in the ideal case (i.e., noise is not present and the parameters of drive and response systems exactly match), the synchronization is achieved exponen- tially fast in the synchronization phases. This requirement implies that, if we use only synchronization phases (i.e., no autonomous phases), then the synchronization is achieved exponentially fast. This requirement is satisfied in most of the synchronization schemes proposed in the literature. This also implies that the error dynamics associated with the difference of the signals of the drive and response systems is exponentially stable. We then showed that, if the synchronization and autonomous phase intervals are chosen appropriately, then the synchronization can be achieved asymptotically. Moreover, the synchronization error decays exponentially to zero (see Theorem 1). We note that the exponential stability is quite important in the robustness of synchronization schemes, and the robustness of many proposed synchronization schemes with respect to noise and parameter mismatch may be considered as a consequence of this property.

We also considered the nonideal case, and showed that the proposed scheme is robust with respect to noise and parameter mismatch. We showed that, if the synchronization and autonomous phase intervals are chosen appropriately, then the synchronization error remains bounded. Moreover, this bound depends linearly on the magnitudes of the noise and the parameter difference (see Theorem 2). We emphasize that Theorem 2 is applicable to any synchronization scheme, provided that exponential synchronization requirement holds and can be considered as a consequence of exponential stability of error dynamics.

Several improvements on the scheme proposed in this paper are possible. The estimates given by Eqs. (14) and (15) appear to be very conservative and may be improved. An optimum relation between $T_{s}$ and $T_{a}$ may also be obtained. An electronic circuit implementation may also be possible (see Ref. [8]). As for any synchronization scheme, our synchronization scheme may also be used for secure communication (see Ref. [14]). Work along these lines is in progress and the results will be presented elsewhere.
[1] L. M. Pecora and T. L. Carroll, Phys. Rev. Lett. 64, 821 (1990).

[2] L. M. Pecora and T. L. Carroll, Phys. Rev. A 44, 2374 (1991).

[3] L. O. Chua, L. Kocarev, and K. Eckert, Int. J. Bifurcation Chaos 2, 705 (1992).

[4] M. J. Ogorzalek, IEEE Trans. Circuits Syst. 40, 693 (1993).

[5] L. Kocarev, K. S. Halle, K. Eckert, and L. O. Chua, Int. J. Bifurc. Chaos 2, 709 (1992).

[6] C. W. Wu and L. O. Chua, Int. J. Bifurc. Chaos 3, 1619 (1993).

[7] K. S. Halle, C. W. Wu, M. Itoh, and L. O. Chua, Int. J. Bifurc. Chaos 3, 469 (1993).

[8] K. M. Cuomo and A. V. Oppenheim, Phys. Rev. Lett. 71, 65 (1993)

[9] K. M. Cuomo, A. V. Oppenheim, and S. H. Strogatz, IEEE
Trans. Circuits Syst. 40, 626 (1993).

[10] L. Kocarev and U. Parlitz, Phys. Rev. Lett. 74, 5028 (1995).

[11] R. E. Amritkar and N. Gupte, Phys. Rev. E 47, 3889 (1993).

[12] Ö. Morgül and E. Solak, Phys. Rev. E 54, 4803 (1996).

[13] Ö. Morgül and E. Solak, Int. J. Bifurc. Chaos (to be published).

[14] Ö. Morgül and M. Feki (unpublishedl).

[15] H. Torikali and T. Saito, IEEE Trans. Circuits Syst. 43, 374 (1996).

[16] M. Vidyasagar, Nonlinear Systems Analysis (Prentice-Hall, Englewood Cliffs, NJ, 1978).

[17] H. K. Khalil, Nonlinear Systems (Macmillan, New York, 1992), pp. 180-208.

[18] W. Hahn, Stability of Motion (Springer-Verlag, New York, 1967). 